\title{
EGFR Mutational Status of Primary Lung Adenocarcinoma in an Indian Cohort Based on 2015 WHO Classification of Lung tumors
}

\author{
Priyanka Yogendra Ravi ${ }^{1 *}$, Anila Korula ${ }^{1}$, Visalakshi Jeyaseelan ${ }^{2}$, Dhananjayan Sakthi ${ }^{1}$ and Rekha Pai ${ }^{1}$ \\ 'Department of General Pathology, Christian Medical College, Vellore, India \\ ${ }^{2}$ Department of Biostatistics, Christian Medical College, Vellore, India
}

\begin{abstract}
Background: Epidermal growth factor receptor (EGFR) mutations have been known to be associated with adenocarcinoma, women, non- smokers and East-Asian ethnicity. This study was aimed to characterize the frequency of EGFR mutations and their association with histologic subtypes in primary lung adenocarcinoma in an Indian cohort.

Methods: Two seventy-four cases were categorized using 2015 WHO classification of lung tumors. The frequency of each histologic subtype and cell type was correlated with EGFR exon sequences in a subset of 120 cases using polymerase chain reaction (PCR) gene sequencing.

Results: The predominant biopsy categories in 274 cases were acinar 167(61\%), solid 63(23\%), mucinous 19(7\%), lepidic 11(4\%) and others 14(5\%). EGFR mutations were detected in 49/120 (40.8\%) including 3/5(60\%) lepidic, 4/9(44.4\%) papillary, 29/68(42.7\%) acinar, 10/24(41.7\%) solid and 1/13(7.7\%) mucinous subtypes and were significantly associated with the cuboidal cell type ( $\mathrm{p}=0.01$ ). These mutations were common in women and non-smokers, although not statistically significant. Exon 19 mutations predominated in 36/49(73.4\%). The majority, 213/263 (81\%), were thyroid transcription factor 1(TTF-1) positive. The polygonal cell type and the solid subtype were frequent amongst stage IV tumors and smokers.
\end{abstract}

Conclusions: EGFR mutations were most frequently seen with the lepidic and papillary subtypes, not associated with the mucinous subtype, more common in women and non-smokers and significantly associated with the cuboidal cell type.

Keywords: EGFR Mutation, Lung Cancer, Histology, Adenocarcinoma, Who Classification, India

\section{Introduction}

Non-small cell lung cancer (NSCLC) accounts for $75-80 \%$ of all lung cancers. Histologically, NSCLC is classified into squamous cell carcinoma, adenocarcinoma, and large cell carcinoma. In India, squamous cell carcinoma has been the most common histological type of NSCLC with a growing predominance of adenocarcinoma ${ }^{[1]}$. Lung adenocarcinomas are well known to display inter and intra-tumoral heterogeneity with profound implications for exact histological classification by pathologistsaccounting for more deaths than breast, prostate and colon cancer combined. ${ }^{[2]}$ While treatment decisions are determined primarily by stage, therapeutically non small cell lung cancer (NSCLC]. The New International classification (IASLC/ATS/ERS) ${ }^{[3,4]}$ and 2015 World Health Organization (WHO) classification ${ }^{[5]}$ "defined the adenocarcinoma in situ(AIS), minimally invasive adenocarcinoma (MIA) and invasive lepidic adenocarcinoma, replaced the 'mixed subtype category' in 2004 WHO classification ${ }^{[6]}$ with the predominant subtype, included the micropapillary subtype and replaced the term "mucinous bronchioloalveolar carcinoma (BAC)" with invasive mucinous adenocarcinoma.

Over the past decade, introduction of targeted therapy with epidermal growth factor receptor- tyrosine kinase inhibitors (EGFR-TKI) has revolutionized the treatment of adenocarcinoma. EGFR activating mutations are associated with clinical phenotype like females, non-smokers, EastAsian ethnicity, and adenocarcinoma, demonstrating responsiveness to tyrosine kinase inhibitors like gefitinib or erlotinib ${ }^{[7]}$. Although a histological correlation has been well established between lung adenocarcinoma and EGFR mutations, there is limited information regarding the correlation between the predominant histological subtype of adenocarcinoma and EGFR mutations in an Indian cohort. Therefore, this analytic and descriptive 
study was aimed at comprehensive histological subtyping and analysis of the frequency of EGFR mutations in primary lung adenocarcinomas based on 2015 WHO classification of lung tumors and correlating this data with clinicopathological features in an Indian cohort.

\section{Materials and Methods}

This retrospective study was conducted in a referral tertiary care center in the Department of Pathology, after the approval from the institutional review board.

Patient Selection and Histologic Analysis: This study evaluated all cases of primary lung adenocarcinomas from June 2011- December 2012, using the archival stained Haematoxylin and Eosin (H\&E) slides and formalin fixed paraffin embedded blocks following strict inclusion and exclusion criteria.

Inclusion Criteria: 1) All cases of primary lung adenocarcinoma biopsied at our institution during June 2011- December 2013.

Exclusion Criteria: 1) Cases with no definite morphological features and/or no immunohistochemical evidence of adenocarcinoma, 2) Squamous cell carcinoma by morphology or by Immunohistochemistry, 3) Non-small cell carcinoma, not otherwise specified (NSCC-NOS); 4) Large cell carcinoma and 5) Inadequate biopsy material for definite categorization.

Cases were categorized using 2015 WHO classification of lung tumors ${ }^{[5]}$ " incorporated from the IASLC/ATS/ ERS classification for small biopsies ${ }^{[3]}$. Data regarding the relevant history, clinical diagnosis, and radiological findings were obtained from hospital records. A detailed histopathological analysis was performed independently by two pathologists (A.K and P.R). Individual features including the predominant cell type, presence of mucin (PAS-Diastase), desmoplasia, stromal elastosis, lymphovascular invasion, and necrosis were studied. Comprehensive histologic subtyping was done to assess histologic patterns semi-quantitatively in $5 \%$ increments, choosing a single predominant pattern. These features were correlated with one or more immunohistochemical markers namely Thyroid Transcription Factor-1 (TTF-1) (Clone 8G7G3-1, Dako, Glostrup, Denmark), cytokeratin 7(CK7) (Clone OV-TL12/30, Dako, Glostrup, Denmark), and Ep-CAM (BerEp4) (Clone Ber-EP4, Dako, Glostrup, Denmark) where available, using the automated Ventana system. CK 7 was used to differentiate between primary and metastatic lung adenocarcinoma, while BerEp4 immunohistochemistry was used to exclude pleural tumors. Details, where available, for stage of disease and smoking status, were included. Patients were staged according to the American Joint Committee on Cancer (AJCC) TNM staging manual, seventh edition ${ }^{[8]}$. EGFR mutational analyses were performed using polymerase chain reaction (PCR) sequencing of exons 18, 19, 20 and 21.

EGFR Mutational Analysis: A subset of 120 cases based on sample size calculation were randomly selected from the pool of cases of primary lung adenocarcinoma for EGFR mutational analysis. EGFR mutational analysis was performed by PCR on formalin fixed and paraffin embedded blocks. The area with tumor cellularity of $>$ $50 \%$ was identified. The tumor was further manually microdissected and 5 paraffin sections at 3-4 $\mu$ m thickness were used for DNA extraction. Extraction was carried out using the DNA tissue extraction kit (QIAGEN India Pvt. Ltd, New Delhi, India). The DNA was quantitated using the Nano-drop (Nano-Drop Technologies, Wilmington, Delaware, USA) and the 260/280 ratio was determined.

Molecular Analysis: The PCR for four exons, namely 18, 19,20 and 21 was performed using the primer sequences by Lynch et al ${ }^{[0] "}$ " (EGFR. All reactions were carried in 25 $\mu 1$ volume. The following thermal cycling profile was used for all PCRs: $95^{\circ} \mathrm{C}$ for $8 \mathrm{~min}, 95^{\circ} \mathrm{C}$ for 30 secs, optimized anneal for $30 \mathrm{secs}, 72^{\circ} \mathrm{C}$ for $1 \mathrm{~min}$ and final extension of $72^{\circ} \mathrm{C}$ for $10 \mathrm{~min}$. The PCR product was detected using a $1.5 \%$ agarose gel. Sequencing of both the sense and antisense strands for all exons was performed with an automated DNA sequencer (ABI PRISM 310 genetic analyzer) using the ABI PRISM Big Dye Terminator Cycle Sequencing Ready Reaction Kit (Applied Biosystems, Foster City, California, USA). Mutational analysis was performed by comparing the sequence with the wild type and by identifying all known mutations in these exons.

Statistical Analysis: Descriptive statistics for continuous data was analyzed using mean with standard deviation or median with inter-quartile range. Categorical data were described using frequencies and percentages. Histopathological subtypes with EGFR mutational analysis were associated using Fischer's exact test and Chi-square test. For all analyses, a $\mathrm{p}<0.05$ was considered statistically significant. Data were analyzed using the SPSS Statistics software, version 16 .

\section{Results}

Patient Characteristics: The study included 274 cases of primary lung adenocarcinoma. Of the 274 cases, 186 $(67.88 \%)$ were males and $88(32.12 \%)$ were females with the mean age being 58 (range 30-76 years) and 55 years (range 32-78 years) respectively. As per the geographical distribution, $148(54 \%)$ were from the east,93 (33.90\%) from the south, $6(2.2 \%)$ from the north, $2(2.2 \%)$ from the west of India. Twenty-five (9.1\%) cases were from Bangladesh. Smoking history was obtained in 165 cases, and a majority of 
patients were non-smokers $117(70.9 \%)$, while $48(29.1 \%)$ were smokers. The commonest location of tumors was the right lung in $125(45.6 \%)$, while in $76(27.7 \%)$ they were bilateral and $69(26.6 \%)$ in the left lung. Although the right lung had a predilection for tumors, the right upper and lower lobes were involved in equal frequencies. The hilum was infrequently involved. The tumor dimension of $>3 \mathrm{~cm}$ documented on CT scan was seen in 178/206 (86.4\%) cases. Amongst 237 cases where staging data were available, a significant proportion $195(82.2 \%)$ were stage IV at diagnosis, 31(13.5\%) were stage III and 11(4.7\%) were Stage II and none with Stage I of disease.

Histologic Findings and Immunohistochemistry: Of the 274 cases, the predominant subtypes were 167 (61\%) acinar, 63(23\%) solid, 19 (7\%) invasive mucinous, 11(4\%) lepidic, $9(3.30 \%)$ papillary, $2(0.7 \%)$ colloid and $2(0.7 \%)$ non-small cell carcinoma, not otherwise specified (NSCCNOS) and 1micropapillary ( $0.3 \%)$ subtype(Figure $1 \mathrm{~A}-1 \mathrm{D}$; Figures 2A-2D).

The polygonal cell type was the most frequent comprising of $160(58.40 \%)$ cases followed by columnar $69(25.2 \%)$, cuboidal 38(13.9\%), signet ring 4 (1.4\%), hobnail $2(0.7 \%)$ and clear cell $1(0.4 \%)$ types. A significant percentage of tumors were associated with mucin production, desmoplasia, stromal elastosis, lymphovascular invasion and necrosis (Table 1). Lymphovascular invasion was most commonly seen amongst stage III/ stage IV disease in $187 / 226(82.7 \%)$. The majority of tumors, 213/263 (81\%) were TTF-1 positive. Almost all cases, demonstrated positivity for CK7 134/135 (99.3\%) and Ber-EP4 63/65 (97\%).

Correlation of Histologic Subtypes with Smoking history: The papillary subtype in 7/7 (100\%) followed by the lepidic predominant in $4 / 5(80 \%)$, acinar in $75 / 102$ (73.6\%) and solid in 16/36 (44.4\%) subtypes were frequent with non-smokers. The solid subtype was relatively more frequent among smokers, $20 / 36(55.6 \%)$ as compared to the other subtypes.

EGFR Mutational Analysis: EGFR mutational analysis was performed on a subset of 120 cases and 49 (40.8\%) were found to harbor a mutation in any one of the four exons $(18,19,20,21)$ that are known to be hotspots of mutations, except one case which had combined exon 20 and 21 mutations. Exon 19 mutations predominated in 36/49 (73.4\%) cases with del E746-A750, as the commonest form with a few other uncommon deletions (Figure 3).There were 12(24.5\%) cases with exon 21 mutation, all with the L858R mutations.

\section{Correlation of EGFR Mutations with Histologic Subtype and Clinicopathological Features:}

A comparison of the mutational pattern with the histological subtype indicated that mutations were most commonly seen in the lepidic subtype $3 / 5(60 \%)$, followed by papillary $4 / 9$ (44.4\%), acinar $29 / 68(42.7 \%)$ and solid subtypes10/24 (41.7\%) in decreasing frequencies. Mucinous adenocarcinomas were infrequently associated with EGFR mutations 1/13(7.7\%), with statistical significance $\mathbf{p}=\mathbf{0} .01$ (Table 2).

Although EGFR mutations were more common amongst females 25/52 (48.1\%) and non-smokers 30/66 (45.5\%), they were also noted amongst smokers 5/18 (27.8\%) and males 22/68 (32.4\%). There was no statistical association of EGFR mutational status with the geographical distribution of patients, residing in India and outside of India. TTF-1 positivity was significantly associated with EGFR mutational status $(\mathbf{p}=\mathbf{0}$.007). Regression analysis of patients with mutation and TTF-1 positivity showed a relative risk of 5. (Table 3)

Correlation of Cell Type with EGFR Mutations, Smoking Status and Stage of Disease: Although the polygonal cell type 21/47(44.6\%) was more frequent with EGFR mutations, there was no statistical significance between the two. However, there was a significant association between the cuboidal cell type and EGFR mutations $(\mathbf{p}=0.01)$. The polygonal cell type was more common in smokers $27 / 48(60.4 \%)$ and in stage IV disease 112/195(57.4\%).

\section{Discussion}

The prevalence of EGFR mutations in this study was $40.8 \%$ similar to a study published from our center, where a prevalence of $39.6 \%$ of EGFR mutations was reported

Table 1: Associated histopathological features with lung adenocarcinoma.

\begin{tabular}{|l|c|c|c|c|}
\hline Associated features & $\mathbf{N}=$ & Present (\%) & Absent (\%) & p value \\
\hline Mucin production & 274 & $226(82.5)$ & $48(17.5)$ & 0.002 \\
\hline Desmoplasia & 274 & $267(97.4)$ & $7(2.6)$ & 0.02 \\
\hline Stromal elastosis & 274 & $252(92)$ & $22(8)$ & 0.05 \\
\hline Lymphovascular invasion & 274 & $227(82.8)$ & $47(17.2)$ & 0.01 \\
\hline Necrosis & 274 & $175(63.9)$ & $99(36.1)$ & 0.18 \\
\hline
\end{tabular}


Table 2:Association of EGFR mutations with predominant histological subtype of lung adenocarcinoma.

\begin{tabular}{|l|c|c|c|c|}
\hline Subtype & Ne & Negative EGFR & Mutated EGFR & p value \\
\hline Lepidic & 5 & $2(40 \%)$ & $3(60 \%)$ & 0.40 \\
\hline Papillary & 9 & $5(55.6 \%)$ & $4(44.4 \%)$ & 0.73 \\
\hline Acinar & 68 & $39(57.3 \%)$ & $29(42.7 \%)$ & 0.34 \\
\hline Solid & 24 & $14(58.3 \%)$ & $10(41.7 \%)$ & $>.99$ \\
\hline Mucinous & 13 & $12(92.3 \%)$ & $1(7.7 \%)$ & 0.01 \\
\hline Micropapillary & 1 & $1(100 \%)$ & - & \\
\hline Abbreviation: EGFR, Epidermal Growth Factor Receptor. & & \\
\hline
\end{tabular}

Table 3: Correlation of EGFR mutational status with clinicopathological features and TTF-1 immunohistochemistry.

\begin{tabular}{|c|c|c|c|c|}
\hline Characteristics & Number (\%) & Wild type (EGFR) & Mutated (EGFR) & $p$ value \\
\hline Location & $\mathrm{N}=120(\%)$ & & & \\
\hline North & $4(3.3 \%)$ & $3(75 \%)$ & $1(25 \%)$ & 0.78 \\
\hline West & $2(1.7 \%)$ & $1(50 \%)$ & $1(50 \%)$ & \\
\hline East & $45(37.5 \%)$ & $30(66.7 \%)$ & $15(33.3 \%)$ & \\
\hline South & $61(50.8 \%)$ & $34(55.7 \%)$ & $27(44.3 \%)$ & \\
\hline Outside India & $8(6.7 \%)$ & $5(62.5 \%)$ & $3(37.5 \%)$ & \\
\hline Gender & $\mathrm{N}=120(\%)$ & & & \\
\hline Male & $68(56.7 \%)$ & $46(67.6 \%)$ & $22(32.4 \%)$ & 0.08 \\
\hline Female & $52(43.3 \%)$ & $27(51.9 \%)$ & $25(48.1 \%)$ & \\
\hline Smoking status & $\mathrm{N}=84(\%)$ & & & \\
\hline Smoker & $18(21.4 \%)$ & $13(72.2 \%)$ & $5(27.8 \%)$ & 0.51 \\
\hline Non-smoker & $66(78.6 \%)$ & $36(54.5 \%)$ & $30(45.5 \%)$ & \\
\hline TTF-1 & $\mathrm{N}=112(\%)$ & & & \\
\hline Positive & $96(85.7 \%)$ & $52(78.8 \%)$ & $44(45.8 \%)$ & 0.01 \\
\hline Negative & $16(14.3 \%)$ & $14(21.2 \%)$ & $2(4.3 \%)$ & \\
\hline \multicolumn{5}{|c|}{ Abbreviations: Epidermal Growth Factor Receptors, TTF: Thyroid Transcription Factor } \\
\hline \multicolumn{2}{|c|}{ IHC: Immunohistochemistry } & & & \\
\hline
\end{tabular}

Table 4: Details of studies correlating EGFR mutations and predominant subtypes.

\begin{tabular}{|l|l|l|l|l|}
\hline Author & Number & TNM stage & Specimen type & Predominant subtype associated with EGFR \\
\hline \multirow{2}{*}{ Motoi et a[ ${ }^{[16]}$} & 100 & Stage I-III & Resections & 1.Papillary 35.1\% (13/37) \\
\cline { 3 - 5 } & & & & 2.Micropapillary (NA) \\
\hline
\end{tabular}




\begin{tabular}{|c|c|c|c|c|}
\hline \multirow{2}{*}{ Yoshizawa et al[17]" } & \multirow{2}{*}{167} & \multirow{2}{*}{ Stage I-III } & \multirow{2}{*}{ Resections } & 1.Lepidic $(71.4 \%)$ \\
\hline & & & & 2.Papilllary $(68.5 \%)$ \\
\hline \multirow{4}{*}{ Sun et al ${ }^{[18]^{\prime \prime}}$} & \multirow{4}{*}{382} & \multirow{4}{*}{ NA } & \multirow{4}{*}{ Resections and biopsies } & 1.Papillary $21 / 30(70 \%)$ \\
\hline & & & & 2.Acinar 98/173 (56.6\%) \\
\hline & & & & 3.MP 2/4(50\%) \\
\hline & & & & 4.Infrequent with mucinous subtype $1 / 3(33.3 \%)$ \\
\hline \multirow{4}{*}{ Present study } & \multirow{4}{*}{120} & \multirow{4}{*}{ Stage III-IV } & \multirow{4}{*}{ Small biopsies } & 1. Lepidic $3 / 5(60 \%)$ \\
\hline & & & & 2. Papillary $4 / 9(44.4 \%)$ \\
\hline & & & & 3.Acinar 10/24(41.7\%) \\
\hline & & & & 4. Infrequent with mucinous subtype $1 / 13(7.7 \%)$ \\
\hline
\end{tabular}

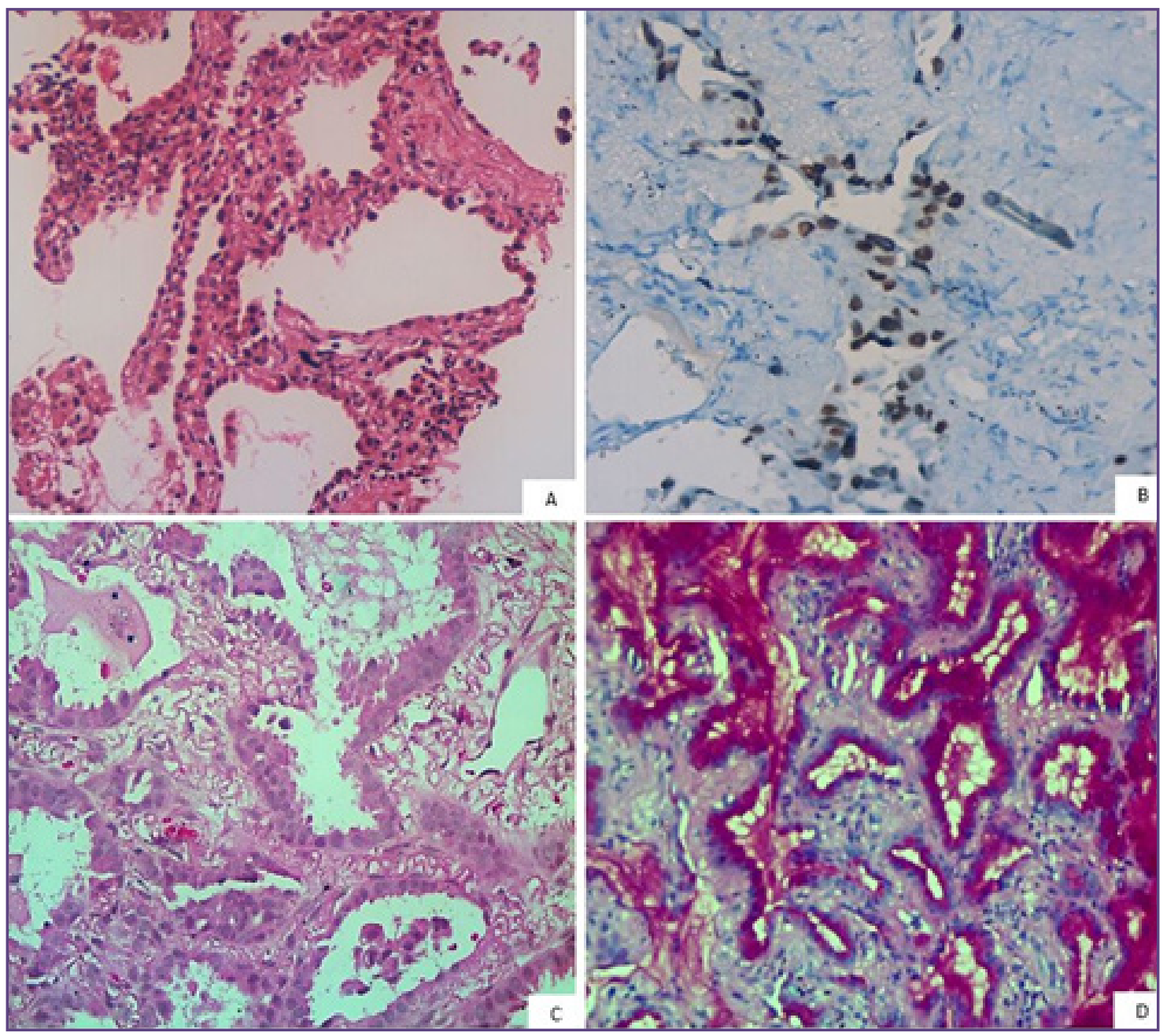

Fig. 1A): Lepidic predominant adenocarcinoma (H\&E,200x); 1B) TTF-1 positive lepidic adenocarcinoma (200x); 1C) Acinar adenocarcinoma with hobnail cell type and elastosis (H\&E 400x); 1D)Invasive mucinous adenocarcinoma (PASD, 200x). 


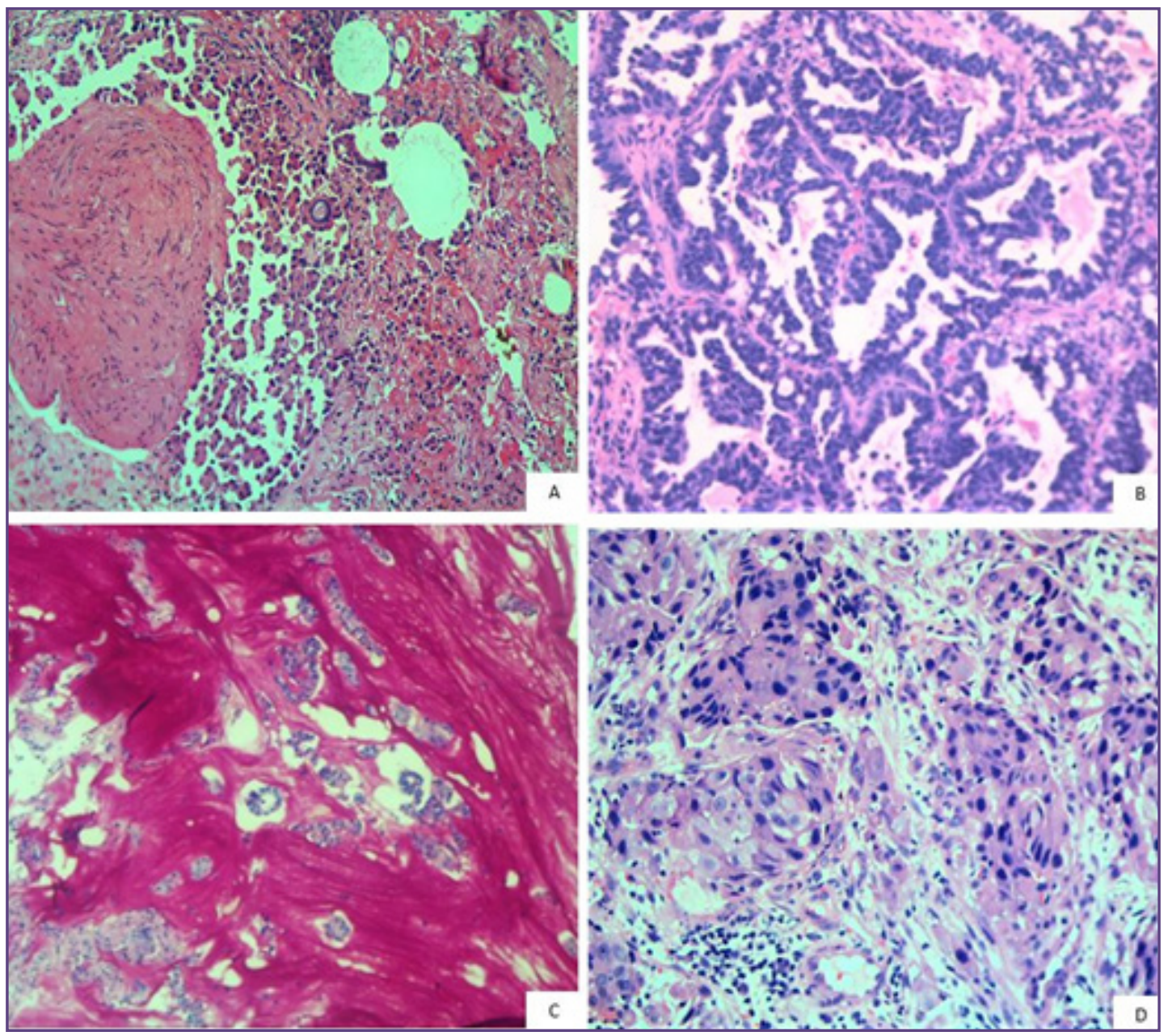

Fig. 2A) Micropapillary adenocarcinoma (H\&E, 200x); 2B) Papillary adenocarcinoma (H\&E, 200x); 2C) Colloid adenocarcinoma (PASD, 200x); 2D) Solid adenocarcinoma (H\&E 200x).

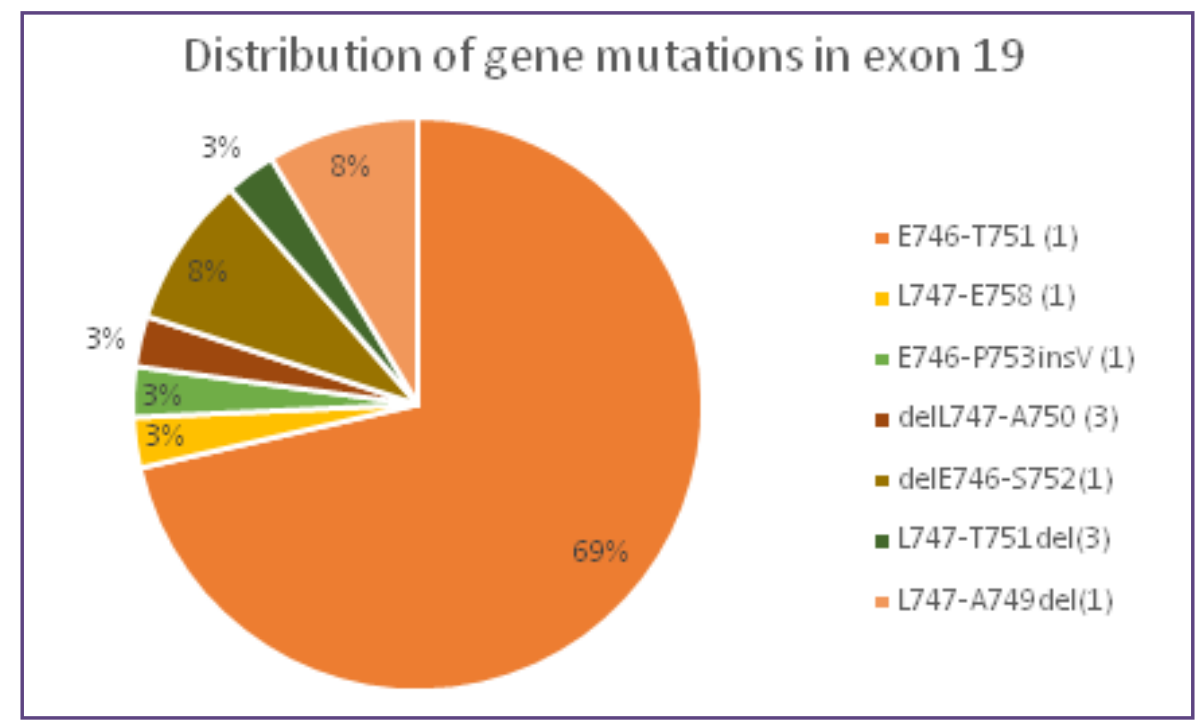

Fig. 3: Distribution of gene mutations in exon 19.

Annals of Pathology and Laboratory Medicine, Vol. 4, Issue 5, September-October, 2017 
with favorableprogression-free survival response with chemotherapy followed by tyrosine kinase inhibitors (TKI's) ${ }^{[10]}$.Studies from India have reported the prevalence of EGFR mutations ranging from $16.6 \%$ - $44 \%^{[10-13]}$. While this fluctuation in values could be attributed to the technique used and the type of histologic diagnosis selected for the study, the role of genetic heterogeneity within India, contributing to variations in mutation frequencies, cannot be entirely ruled out ${ }^{[14]}$.

In the present study, there was a predominance of exon 19 mutations in $73.4 \%$ cases, with 15 bp del (E746-A750) being the commonest form amongst exon 19 mutations(69.4\%). Exon 21 mutations were seen in $24.4 \%$, all being the L858R type. Exon 18 mutation was not seen in any of the cases. Molecular analysis has shown that short in-frame deletions of exon 19 and a point mutation in exon 21 at base pair 2573 , resulting in the substitution of leucine by arginine in codon 858 (L858R) make up for $\sim 90 \%$ of all mutations. Point mutations in exons 18 and exon 20 account for 5\% of EGFR mutations in non-small cell lung cancer ${ }^{[15]}$. In the present study, there was one case with a combined exon 20(T790M) and 21(L858R) mutation. T790M mutations can occur in $50-60 \%$ patients with resistance to TKI's, largely due to a conformational change in the EGFR molecule that prevents drug binding. It is also reported that the $7790 \mathrm{M}$ mutation may confer primary resistance to TKI's and possibly co-exist with L858R mutations ${ }^{[15]}$. This finding was noted in the present study. The study did not find any significant association between mutational status and age, gender, smoking status and TTF-1 positivity.

Several studies have shown that EGFR mutations are associated with the lepidic, papillary, micropapillary and acinar subtypes (Table 4). In the present study, EGFR mutations were most common in the lepidic subtype (60\%) followed by the papillary (44.4\%), and acinar subtypes $(42.7 \%)$ and were rare with the invasive mucinous subtype (7.7\%). These findings are consistent with the reports of Motoi et a ${ }^{[16]}$, Yoshizawa et $\mathrm{a}^{\left[{ }^{17]}\right]}$ and Sun et al ${ }^{[18]}$. We did not find an association between EGFR mutations and the micropapillary subtype, probably due to a very small sample number of this particular subtype. Subtyping of tumors in patients undergoing complete resection, using the WHO 2015 classification appears to be very important, as highlighted by Tsao and colleagues since some subtypes like the solid and micropapillary tumors that have a poorer survival would significantly benefit from adjuvant chemotherapy. In contrast, this may not be as beneficial for acinar and papillary predominant tumors, that tend to have a better survival ${ }^{[19]}$ and subtyping tumors might be able to guide therapy.
In this study, EGFR mutations were more common in females $(48.1 \%)$ and non-smokers $(45.5 \%)$, although a proportion of smokers $(27.8 \%)$ and males $(32.4 \%)$ also were positive for these. Similar results were reported by Sun et $\mathrm{al}^{[18]}$, where the incidence of EGFR mutations in men who smoked with adenocarcinomas was $29.7 \%$ $(35 / 118)$. As per the study by D'Angelo and colleagues in 2,142 patients with lung adenocarcinoma, 57\% of EGFR mutations would be missed if testing were restricted to women who never smoked cigarettes ${ }^{[20]}$. In the present study, we found the solid predominant subtype was more common among smokers, which is similar to the finding by Motoi et al ${ }^{[16]}$.

The current set guidelines recommend mutational analysis of all lung tumors with a diagnosis of adenocarcinoma or with an adenocarcinoma component, irrespective of the age, gender, ethnicity, smoking status, and histological subtype. In a setting of small biopsies and cytology specimens where an adenocarcinoma component cannot be completely excluded, testing for EGFR mutations can be considered in cases showing a small cell or squamous histology. However, in this scenario, clinical criteria like female gender, the absence of smoking history and age, may be useful indicators in the selection of a subset for testing ${ }^{[21]}$.

In the present study, EGFR mutations were significantly more common in tumors expressing TTF-1, hence TTF1 positivity could be used as a predictive marker for EGFR mutations as shown in several other studies ${ }^{[18,22]}$. By regression analysis, cases that were positive for TTF1 were 5 times more likely to harbor an EGFR mutation. However, there was no statistical significance established with TTF-1 and any particular histological subtype.

We also compared the predominant cell type with EGFR mutations, smoking status, and stage of the disease. Although the polygonal cell type was the commonest, the cuboidal cell type was significantly associated with EGFR mutations. A similar study by Okada et al ${ }^{[23]}$ proposed the five-cell type classification and found the hobnail cell type to be significantly associated with EGFR mutations $(\mathrm{p}<0.001)$, followed by mixed, columnar/ cuboidal, polygonal and goblet cells. The study also found a higher percentage of smokers to be significantly associated with the cuboidal/ columnar and polygonal cell type, similar to findings in this study where the polygonal cell type was associated with smokers.

Although survival analysis was not done in the present study, it is worthwhile to note that several clinicopathological parameters have been associated with poor disease-free 
survival, which includes male gender, gross and invasive tumor component, vascular invasion, necrosis and higher stage of disease. The AIS and MIA have a 100\% diseasefree survival (DFS) at 5yrs, followed by the lepidic, acinar and papillary subtypes with a 5-year DFS between $83-90 \%$. The solid, micropapillary, colloid and invasive mucinous subtypes that belong to the poor prognostic group have a 5 -year DFS between $67-76 \%{ }^{[24]}$.

A limitation of this study is that the histologic and molecular profile have not been correlated with progression-free survival and overall survival, as was done previously for a cohort of 106 cases at our center ${ }^{[9]}$.

\section{Conclusion}

This study has helped to highlight that EGFR mutations were most frequently seen with the lepidic and papillary subtypes, not associated with the mucinous subtype, were more common in women and non-smokers and were significantly associated with the cuboidal cell type. This data will be of value to overcome the lacunae in an Indian setting.

\section{Acknowledgments}

Department of General Pathology, Christian Medical College, Vellore, India.

\section{References}

1. Behera D. Epidemiology of lung cancer-Global and Indian perspective. J Indian Acad Clin Med. 2012;13:131-7.

2. Pikor LA, Ramnarine VR, Lam S, Lam WL. Genetic alterations defining NSCLC subtypes and their therapeutic implications. Lung Cancer Amst Neth. 2013 Nov;82(2):179-89.

3. Travis WD, Brambilla E, Noguchi M, Nicholson AG, Geisinger K, Yatabe Y, et al. Diagnosis of Lung Cancer in Small Biopsies and Cytology: Implications of the 2011 International Association for the Study of Lung Cancer/American Thoracic Society/European Respiratory Society Classification. Arch Pathol Lab Med. 2013 May;137(5):668-84.

4. Travis WD, Brambilla E, Noguchi M, Nicholson AG, Geisinger K, Yatabe Y, et al. Diagnosis of Lung Adenocarcinoma in Resected Specimens: Implications of the 2011 International Association for the Study of Lung Cancer/American Thoracic Society/European Respiratory Society Classification. Arch Pathol Lab Med. 2013 May;137(5):685-705.

5. Travis WD, Brambilla E, Nicholson AG, Yatabe Y, Austin JHM, Beasley MB, et al. The 2015 World Health Organization Classification of Lung Tumors. J Thorac Oncol. 2015 Sep 1;10(9):1243-60.
6. Travis WD. Pathology \& Genetics: Tumours of the Lung, Pleura, Thymus, and Heart [Internet]. Vol. 7. Iarc; 2004 [cited 2013 Nov 15].

7. Tsao M-S, Sakurada A, Cutz J-C, Zhu C-Q, Kamel-Reid $\mathrm{S}$, Squire J, et al. Erlotinib in lung cancer-molecular and clinical predictors of outcome. $\mathrm{N}$ Engl $\mathrm{J}$ Med. 2005;353(2):133-144.

8. Edge SB, Byrd DR, Compton CC, Fritz AG, Greene FL, Trotti A, et al. AJCC cancer staging manual. 2010. Vol 17th Ed N Y Springer. 2010;

9. Lynch TJ, Bell DW, Sordella R, Gurubhagavatula S, Okimoto $\mathrm{RA}$, Brannigan BW, et al. Activating mutations in the epidermal growth factor receptor underlying responsiveness of non-small-cell lung cancer to gefitinib. N Engl J Med. 2004 May 20;350(21):2129-39.

10. Bhatt AD, Pai R, Rebekah G, Nehru GA, Dhananjayan S, Samuel A, et al. Clinicopathologic features of non-small cell lung cancer in India and correlation with epidermal growth factor receptor mutational status. Indian J Cancer. 2013 Jun;50(2):94-101.

11. Maturu VN, Singh N, Bal A, Gupta N, Das A, Behera D. Relationship of epidermal growth factor receptor activating mutations with histologic subtyping according to International Association for the Study of Lung Cancer/ American Thoracic Society/European Respiratory Society 2011 adenocarcinoma classification and their impact on overall survival. Lung India Off Organ Indian Chest Soc. 2016;33(3):257.

12. Sahoo R, Harini VV, Babu VC, Patil Okaly GV, Rao S, Nargund A, et al. Screening for EGFR mutations in lung cancer, a report from India. Lung Cancer Amst Neth. 2011 Sep;73(3):316-9.

13. Veldore VH, Rao RM, Kakara S, Pattanayak S, Tejaswi $\mathrm{R}$, Sahoo R, et al. Epidermal growth factor receptor mutation in non-small-cell lung carcinomas: a retrospective analysis of 1036 lung cancer specimens from a network of tertiary cancer care centers in India. Indian J Cancer. 2013 Jun;50(2):87-93.

14. Moorjani P, Thangaraj K, Patterson N, Lipson M, Loh P-R, Govindaraj P, et al. Genetic evidence for recent population mixture in India. Am J Hum Genet. 2013;93(3):422-438.

15. Raparia K, Villa C, DeCamp MM, Patel JD, Mehta MP. Molecular profiling in non-small cell lung cancer: a step toward personalized medicine. Arch Pathol Lab Med. 2013 Apr;137(4):481-91.

16. Motoi N, Szoke J, Riely GJ, Seshan VE, Kris MG, Rusch VW, et al. Lung adenocarcinoma: modification of the 2004 WHO mixed subtype to include the major histologic subtype suggests correlations between papillary and micropapillary 
adenocarcinoma subtypes, EGFR mutations and gene expression analysis. Am J Surg Pathol. 2008;32(6):810.

17. Yoshizawa A, Sumiyoshi S, Sonobe M, Kobayashi M, Fujimoto M, Kawakami F, et al. Validation of the IASLC/ ATS/ERS lung adenocarcinoma classification for prognosis and association with EGFR and KRAS gene mutations: analysis of 440 Japanese patients. J Thorac Oncol Off Publ Int Assoc Study Lung Cancer. 2013 Jan;8(1):52-61.

18. Sun P-L, Seol H, Lee HJ, Yoo SB, Kim H, Xu X, et al. High incidence of EGFR mutations in Korean men smokers with no intratumoral heterogeneity of lung adenocarcinomas: correlation with histologic subtypes, EGFR/TTF-1 expressions, and clinical features. J Thorac Oncol Off Publ Int Assoc Study Lung Cancer. 2012 Feb;7(2):323-30.

19. Tsao M-S, Marguet S, Le Teuff G, Lantuejoul S, Shepherd FA, Seymour L, et al. Subtype classification of lung adenocarcinoma predicts benefit from adjuvant chemotherapy in patients undergoing complete resection. J Clin Oncol. 2015;33(30):3439-3446.

20. D'Angelo SP, Pietanza MC, Johnson ML, Riely GJ, Miller VA, Sima CS, et al. Incidence of EGFR exon 19 deletions and L858R in tumor specimens from men and cigarette smokers with lung adenocarcinomas. J Clin Oncol. 2011;29(15):2066-2070.
21. Lindeman NI, Cagle PT, Beasley MB, Chitale DA, Dacic S, Giaccone $\mathrm{G}$, et al. Molecular testing guideline for selection of lung cancer patients for EGFR and ALK tyrosine kinase inhibitors: guideline from the College of American Pathologists, International Association for the Study of Lung Cancer, and Association for Molecular Pathology. J Thorac Oncol. 2013;8(7):823-859.

22. Leary AF, de Castro DG, Nicholson AG, Ashley S, Wotherspoon A, O'Brien ME, et al. Establishing an EGFR mutation screening service for non-small cell lung cancerSample quality criteria and candidate histological predictors. Eur J Cancer. 2012;48(1):61-67.

23. Okada A, Shimmyo T, Hashimoto T, Kobayashi Y, Miyagi Y, Ishikawa Y, et al. Predictive advantage of a cell type classification for pulmonary adenocarcinoma coupled with data for p53, K-ras and EGFR alterations. Cancer Sci. 2010 Jul 1;101(7):1745-53.

24. Yoshizawa A, Motoi N, Riely GJ, Sima CS, Gerald WL, Kris MG, et al. Impact of proposed IASLC/ATS/ERS classification of lung adenocarcinoma: prognostic subgroups and implications for further revision of staging based on analysis of 514 stage I cases. Mod Pathol Off J U S Can Acad Pathol Inc. 2011 May;24(5):653-64.

*Corresponding author:

Priyanka Y Ravi, 5681 Rhuland street, APT 706, Halifax, Nova Scotia, Canada B3H4J6

Phone: +91 902-210-2222

Email: priyankayravi@gmail.com

Date of Submission : 10.03.2017

Date of Acceptance : 24.06.2017

Financial or other Competing Interests: None. 